\title{
Molecular Diagnosis of Monogenic Diabetes and Clinical/Laboratory Features in Turkish Children
}

\author{
(1) Damla Gökşen ${ }^{1}$, (1) Ediz Yeşilkaya2 , (1) Samim Özen ${ }^{1}$, (1) Yılmaz Kor ${ }^{3}$, (1) Erdal Eren ${ }^{4}$, (1) Özlem Korkmaz 5, (1) Merih Berberoğlu6, \\ (D) Gülay Karagüzel7, (1) Eren Er¹, (D) Ayhan Abacı8, (D) Olcay Evliyaoğlu9 ${ }^{9}$, (D) Emine Demet Akbaş3, (D) Edip Ünal10, (D) Semih Bolu11, \\ (D) Özlem Nalbantoğlu12, (D) Ahmet Anık13, (D) Meltem Tayfun¹4, (D) Muammer Büyükinan15, (D) Saygın Abalı16, (D) Gülay Can Yılmaz¹, \\ (D) Deniz Kör18, (D) Elif Söbü19, (D) Zeynep Şıklar6, (D) Recep Polat20, (D) Şükran Darcan1 \\ ${ }^{1}$ Ege University Faculty of Medicine, Department of Pediatric Endocrinology, Izmir, Turkey \\ 219 Mayıs Hospital, Clinic of Pediatric Endocrinology, Ankara, Turkey \\ ${ }^{3}$ Adana City Training and Research Hospital, Clinic of Pediatric Endocrinology, Adana, Turkey \\ ${ }^{4}$ Bursa Uludağ University Faculty of Medicine, Department of Pediatric Endocrinology, Bursa, Turkey \\ ${ }^{5}$ Başkent University Faculty of Medicine, Department of Pediatric Endocrinology, Ankara, Turkey \\ ${ }^{6}$ Ankara University Faculty of Medicine, Department of Pediatric Endocrinology, Ankara, Turkey \\ ${ }^{7}$ Karadeniz Technical University Faculty of Medicine, Department of Pediatric Endocrinology, Trabzon, Turkey \\ ${ }^{8}$ Dokuz Eylül University Faculty of Medicine, Department of Pediatric Endocrinology, İzmir, Turkey \\ 9 Iistanbul University, İstanbul Faculty of Medicine, Department of Pediatric Endocrinology, Istanbul, Turkey \\ ${ }^{10}$ Dicle University Faculty of Medicine, Department of Pediatric Endocrinology, Diyarbakır, Turkey \\ ${ }^{11}$ Adıyaman University Training and Research Hospital, Clinic of Pediatric Endocrinology, Adıyaman, Turkey \\ 12 University of Health Sciences Turkey, Dr. Behçet Uz Children's Diseases and Surgery Training and Research Hospital, Clinic of Pediatric \\ Endocrinology, İmir, Turkey \\ ${ }^{13}$ Aydın Adnan Menderes University Faculty of Medicine, Department of Pediatric Endocrinology, Aydın, Turkey \\ 14Lokman Hekim University Faculty of Medicine, Department of Pediatric Endocrinology, Ankara, Turkey \\ ${ }^{15}$ Konya Training and Research Hospital, Clinic of Pediatric Endocrinology, Konya, Turkey \\ ${ }^{16}$ Acıbadem University Faculty of Medicine, Department of Pediatric Endocrinology, Istanbul, Turkey \\ ${ }_{17}$ Mardin Public Hospital, Clinic of Pediatric Endocrinology, Mardin, Turkey \\ ${ }^{18}$ Çukurova University Faculty of Medicine, Department of Pediatrics Nutrition and Metabolic Diseases, Adana, Turkey \\ 19istanbul Kartal Dr. Lütfi Kırdar City Hospital, Clinic of Pediatric Endocrinology, İstanbul, Turkey \\ 20Sakarya Training and Research Hospital, Clinic of Pediatric Endocrinology, Sakarya, Turkey
}

\section{What is already known on this topic?}

Monogenic diabetes is a heterogeneous group of disorders, accounting for just 1-6\% of all diabetes. Variants in $H N F 1 A$, $H N F 4 A$, and GCK accounts for most MODY-monogenic diabetes cases. Patient numbers and information are limited on less common causes of monogenic forms of diabetes.

What this study adds?

This study is the first Turkish multicenter genetic study of patients with molecularly diagnosed monogenic diabetes. This study determined the clinical and laboratory features, the admission characteristics and distribution of monogenic diabetes in childhood. The distribution in this cohort was in disagreement with the previously published distribution.

Address for Correspondence: Damla Gökșen MD, Ege University Faculty of Medicine, Department of Pediatric Endocrinology, İzmir, Turkey

Phone: +902323901230 E-mail: damla.goksen@ege.edu.tr ORCID: orcid.org/0000-0001-6108-0591
Conflict of interest: None declared Received: 07.03.2021 Accepted: 08.07.2021

${ }^{\circ}$ Copyright 2021 by Turkish Pediatric Endocrinology and Diabetes Society

The Journal of Clinical Research in Pediatric Endocrinology published by Galenos Publishing House. 


\section{Abstract}

Objective: Monogenic diabetes is a heterogeneous disease that causes functional problems in pancreatic beta cells and hyperglycemia. The aim of this study was to determine the clinical and laboratory features, the admission characteristics and distribution of monogenic form of diabetes in childhood in Turkey.

Methods: Patients aged 0-18 years, who were molecularly diagnosed with monogenic diabetes, and consented to participate, were included in the study.

Results: Seventy-seven (45.6\%) female and 92 male cases with a mean age of $8.18 \pm 5.05$ years at diagnosis were included. $52.7 \%$ of the cases were diagnosed with monogenic diabetes by random blood glucose measurement. The reason for genetic analysis in $95(56.2 \%)$ of cases was having a family member diagnosed with diabetes under the age of 25 . At the time of diagnosis, ketone was detected in urine in $16.6 \%$ of the cases. Mean hemoglobin A1c on admission, fasting blood glucose, fasting insulin, and c-peptide values were $7.3 \pm 2.1 \%, 184.9 \pm 128.9 \mathrm{mg} / \mathrm{dL}, 9.4 \pm 22.9 \mathrm{IU} / \mathrm{L}, 1.36 \pm 1.1$ and $\mathrm{ng} / \mathrm{L}$ respectively. GCK-MODY was found in 100 (59.2\%), HNF1 AMODY in 31 (18.3\%), and variants in ABCC8 in 6 (3.6\%), KCNJ11 in 5 (3\%), HNF4A in 2 (1.2\%), and HNF1B in 2 (1.2\%).

Conclusion: Recent studies have indicated HNF1 A-MODY is the most frequent of all the MODY-monogenic diabetes cases in the literature $(50 \%)$, while GCK-MODY is the second most frequent (32\%). In contrast to these reports, in our study, the most common form was GCKMODY while less than $20 \%$ of cases were diagnosed with HNF1A-MODY.

Keywords: Monogenic diabetes, early-onset diabetes, next-generation sequencing, GCK, HNF1A

\section{Introduction}

Monogenic diabetes is a monogenic, clinically and genetically heterogeneous form of diabetes. This includes neonatal diabetes mellitus, maturity onset diabetes of the young (MODY) and rare diabetes-associated syndromes due to defects in beta cell function. The disease may be inherited within families as a dominant, recessive, or nonMendelian trait or may present as a spontaneous case due to a de novo variant. Well over 40 different genetic subtypes of monogenic diabetes have been identified to date, each having a typical phenotype and a specific pattern of inheritance (1). The acronym MODY was used to highlight its firm hereditary basis (2).

MODY is estimated to account for only about $1-2 \%$ of all cases attributed to diabetes, and is usually initially misclassified as type 1 or type 2 diabetes $(3,4)$. Previously, 13 MODY subtypes were identified, and recently a $14^{\text {th }}$ subtype, which is caused by a heterozygous variant in the APPL1 gene, has been added to the list. All subtypes have in common a primary defect in insulin secretion associated with pancreatic beta cell dysfunction (5). Heterozygous variants in the GCK (glucokinase) (MODY 2), HNF1A (hepatocyte nuclear factor 1 alpha) (MODY 3), and HNF4A (hepatocyte nuclear factor 4 alpha) (MODY 1) genes are the most frequent, and together they account for over $95 \%$ of the known genetic causes of MODY. The relative frequencies of MODY subtypes show variations across countries because of the use of different selection criteria for patients for genetic testing (6).

The aim of the study was to determine the clinical and laboratory features, the admission characteristics, and distribution of monogenic diabetes in childhood in Turkey.

\section{Methods}

\section{Subjects}

In this multicenter genetic study, the data of participants were cross-sectionally analyzed. A nation-wide, webbased, CEDD-NET Data System (http://cedd.saglik-network. org/) was used for data collection between March 2016 and April 2017. Written informed consent was obtained from all subjects, and the study was approved by Gülhane Military Medical Academy, Ethics Committee on 17.02.2016 (number: 50687469-1491-191-16/1648-409).

A total of 169 patients, aged 0-18 years, who were molecularly diagnosed with monogenic diabetes were enrolled. The suspected clinical diagnoses of monogenic diabetes were based on: (1) The early onset of diabetes ( $<25$ years of age); (2) negative pancreatic autoantibodies; (3) persistently detectable C-peptide; and (4) low or no insulin requirement 3 years after the diagnosis or dominant inheritance or incidental hyperglycemia.

Clinical data of the patients, including gender, age at diagnosis, and family history of diabetes such as diabetic complications and treatments, were obtained through medical records during hospitalization. Laboratory data at diagnosis, including blood glucose, insulin, C-peptide, glycated hemoglobin A1c (HbA1c), and autoantibodies were also collected.

\section{Genetic Studies}

To confirm the diagnosis of monogenic diabetes, genetic tests were performed in various genetic laboratories and entered onto the statistics program by each center participating in 
the study. Sequence variants were analyzed by VarSome (7), and classified according to the American College of Medical Genetics and Genomics (ACMG) guidelines (8). Variants evaluated as "pathogenic" and "likely pathogenic" according to ACMG criteria were included in the study.

\section{Statistical Analysis}

Data analysis was performed with IBM-Statistical Package for the Social Sciences for Windows, version 21.0, software (IBM Inc., Armonk, NY, USA). Descriptive statistics of the clinical and laboratory findings were expressed as mean \pm standard deviation, number, and percentage. Since the number of patients in most subgroups were few and not homogeneously distributed, comparative statistics were not performed in order to avoid type 2 statistical errors.

\section{Results}

A total of 169 patients, 92 males $(54.4 \%)$ and 77 females $(45.6 \%)$, were included in the study. The mean age of the patients at diagnosis was $8.18 \pm 5.05$ years. The mean birth weight was $3110 \pm 660 \mathrm{~g}$ and the mean gestation week was $38.5 \pm 2.1$ weeks. Presenting complaints included: incidental diagnosis in 89 patients $(52.7 \%$ ); polyuria and polydipsia in 50 (29.6\%); family history of diabetes in 14 (8.3\%); weight loss in 5 (3\%); and obesity in $2(1.2 \%)$. Five patients $(3 \%)$ were diagnosed with ketoacidosis and 12 patients $(7.1 \%)$ with ketosis at initial evaluation. No patient had additional features, such as renal cysts.

Initial treatment consisted of diet alone in 93 patients (55\%), diet and insulin treatment in $62(36.7 \%)$, and diet and an oral anti-diabetic drug in $13(7.7 \%)$. Oral anti-diabetic drugs given to the patients at their follow-ups included; sulfonylurea in $34(20.1 \%)$, metformin in 10 (5.9\%), and one $(0.6 \%)$ received repaglinide.

\section{Genetic Findings}

The distribution of the variants was: GCK (MODY 2) in 100 patients (59.2\%); HNF1A (MODY 3) variants in 31 patients $(18.3 \%)$; $A B C C 8$ variants in 6 patients $(3.6 \%)$; KCNJ11 variants in 5 patients (3\%); INS in 4 patients $(2.4 \%)$; HNF $4 A$ (MODY 1 ) in 2 patients (1.2\%); BLK (MODY 11) in 2 patients (1.2\%); HNF1B (MODY 5) in 2 patients (1.2\%); NEUROD1 (MODY 6) in one patient; and CEL (MODY 8) in one patient. Genetic diagnosis was not specified in 15 cases and further genetic analysis is planned (Figure 1).

The reason for requesting genetic analysis in 95 (56.2\%) of the cases was having a family member diagnosed with diabetes under the age of 25 , while in $89(52.2 \%)$ of the cases was autoantibody negativity at the time of diagnosis,

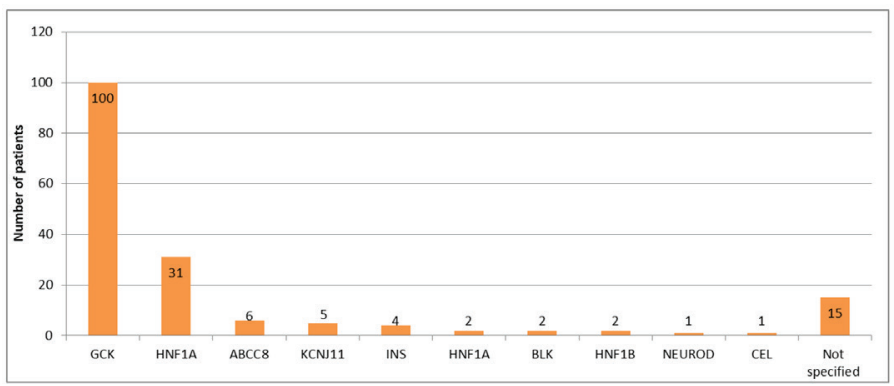

Figure 1. The distribution of the variants causing monogenic forms of diabetes

and in 15 (8.9\%) was that diabetes was diagnosed before 6 months of age.

\section{Characteristics of Patients with GCK Variants}

In patients with a GCK variant (41 females and 59 male), mean age at diagnosis was $8.55 \pm 4.66$ years, mean birth weight was $3160 \pm 460 \mathrm{~g}$, and mean gestation week was $38.9 \pm 1.2$. Anthropometric data and laboratory characteristics of GCK-MODY patients at baseline are summarized in Table 1. Almost $67 \%$ of the cases with GCK-MODY were diagnosed by random blood glucose measurement. None of the patients had ketonemia/ketonuria, ketoacidosis, or dyslipidemia at the time of diagnosis or during follow-up. Of all the patients, 91 had impaired fasting glycemia (IFG), and nine had impaired glucose tolerance (IGT). Forty (40\%) of the patients had typical family history of diabetes in three generations. Sixty-two (62\%) of the patients' parents had been diagnosed before the age of 25 years. Nineteen (19\%) of the patients' mothers were diagnosed during pregnancy. Initial treatment consisted of diet alone in 74 patients $(74 \%)$, diet and insulin treatment in $19(19 \%)$, and diet and an oral anti-diabetic drug in 7 (7\%). Oral anti-diabetic drugs were started in patients at their follow-ups including sulfonylurea in 13 patients $(13 \%)$ and metformin in $5(5 \%)$.

\section{Characteristics of Patients with HNF1A Variants}

In patients with a HNF1A variant, including 17 females $(54.8 \%)$ and 14 males, the mean age at diagnosis was $10.1 \pm 3.6$ years, mean birth weight was $2870 \pm 630 \mathrm{~g}$, and mean gestational week at birth was $38.4 \pm 2.52$. Anthropometric data and laboratory characteristics of HNF1 A-MODY patients at baseline are summarized in Table 2. Nine (29\%) of the patients had ketonemia/ketonuria, but none of the patients had ketoacidosis at the time of diagnosis or during follow-up. Only $6 \%$ of the cases were diagnosed with HNF1A-MODY by random blood glucose measurement. Thirteen (54.8\%) of the patients had typical family history of diabetes in three generations. Twenty-three (74.2\%) parents had been diagnosed before the age of 25 


\begin{tabular}{ll}
\hline Table 1. Anthropometric and laboratory characteristics of \\
GCK-MODY patients $(\mathbf{n}=\mathbf{1 0 0})$ \\
\hline Age at time of study, (years) & $12.24 \pm 5.29$ \\
Age at the time of diagnosis, (years) & $8.55 \pm 4.66$ \\
Female/male, (n) & $41 / 59$ \\
Body mass index $\left(\mathrm{kg} / \mathrm{m}^{2}\right)$ & $17.3 \pm 3.31$ \\
Birth weight, (g) & $3160 \pm 467$ \\
Gestational age at birth, (weeks) & $38.9 \pm 1.2$ \\
Fasting blood glucose, (mg/dL) & $127.8 \pm 30.7$ \\
Fasting insulin, (IU/L) & $8.95 \pm 24.15$ \\
HbA1c, (\%) & $6.40 \pm 0.48$ \\
C-peptide at the time of diagnosis, (ng/mL) & $1.39 \pm 1.05$ \\
\hline HbA1c: hemoglobin A1c
\end{tabular}

\begin{tabular}{ll}
\hline Table 2. Anthropometric and laboratory characteristics of \\
HNF1 A-MODY patients $(\mathbf{n}=\mathbf{3 1})$ \\
\hline Age at time of study, (years) & $14.04 \pm 3.43$ \\
Age at the time of diagnosis, (years) & $10.1 \pm 3.6$ \\
Female/male, (n) & $17 / 14$ \\
Body mass index $\left(\mathrm{kg} / \mathrm{m}^{2}\right)$ & $19.56 \pm 4.15$ \\
Birth weight, (g) & $2870 \pm 630$ \\
Gestational age at birth, (weeks) & $38.4 \pm 2.52$ \\
Fasting blood glucose, (mg/dL) & $218 \pm 111$ \\
Fasting insulin, (IU/L) & $10.5 \pm 7.3$ \\
HbA1c, (\%) & $8.5 \pm 2.5$ \\
C-peptide at the time of diagnosis (ng/mL) & $1.61 \pm 0.99$ \\
\hline HbA1c: hemoglobin A1c & \\
\hline
\end{tabular}

years. Five mothers were diagnosed during pregnancy. Initial treatment consisted of diet alone in 9 patients (29\%), diet and insulin treatment in 19 (61.3\%), and diet and an oral anti-diabetic drug in 3 (9.7\%). Oral anti-diabetic drugs were initiated during patients' follow-ups including sulfonylurea in 34 patients $(20.1 \%)$, metformin in $10(5.9 \%)$, and one $(0.6 \%)$ received repaglinide.

\section{Characteristics of Patients with Variants in Other Genes}

In the present study, $A B C C 8$ variants were found in 6 patients $(3.6 \%)$, KCNJ11 variants in 5 patients $(3 \%)$, INS in 4 patients $(2.4 \%)$, HNF4A (MODY 1 ) in 2 patients (1.2\%), BLK (MODY 11) in 2 patients (1.2\%), HNF1B (MODY 5) in 2 patients (1.2\%), NEUROD1 (MODY 6) in one patient, and CEL (MODY 8) in one patient. The genetic diagnosis was not specified in 15 cases.

The reason for requesting genetic analysis in patients with $A B C C 8$ variants was: being diagnosed before 6 months of age in three cases (50\%); having a family member diagnosed with diabetes under the age of 25 in two cases; and diagnosis of non-obese type 2 diabetes in one. Two of the patients had diabetic ketosis at initial evaluation, while the other four did not. The aforementioned two were on sulphonylurea therapy, while the other four continued on the prior insulin regimen.

The reason for requesting genetic analysis in all the patients with KCJN11 variants was because the patients were diagnosed before 6 months of age. Two of these patients were diagnosed with diabetic ketoacidosis. Sulfonylurea treatment was administered to all the patients and the treatments were continued during their follow-up.

The reason for requesting genetic analysis in INS was due to diabetes being diagnosed before 6 months of age. Three of these patients were diagnosed with diabetic ketosis, and all these patients were given insulin and continued this therapy. The patients with HNF4A (MODY 1) were diagnosed with IGT without ketone in urine. After the genetic results of the patients were obtained, the initial insulin therapy was terminated, and oral anti-diabetic treatment was initiated. The two patients with BLK (MODY 11) were diagnosed with diabetes due to polyuria and polydipsia. One of the patients had diabetic ketosis and, therefore, no response to sulfonylurea treatment so the initial treatment was replaced with insulin therapy. The other patient with the $B L K$ variant had no ketosis and responded to sulfonylurea treatment. One of the two patients with HNF1B variant was diagnosed with diabetes due to polyuria and polydipsia at the age of 12.7 years with an HbA1c of $6.8 \%$ and insulin treatment was deemed unnecessary during the follow-up. As for the other patient who was diagnosed with diabetes due to polyuria and polydipsia with ketosis at the age of 8.1 years and an $\mathrm{HbA} 1 \mathrm{c}$ of $11.2 \%$, insulin therapy was necessary throughout the follow-up period. The patient with NEUROD1 (MODY 6) had the initial complaint of polyuria with IFG. BMI of the patient was $18.51 \mathrm{~kg} / \mathrm{m}^{2}$ and $\mathrm{HbA} 1 \mathrm{c}$, at the time of diagnosis, was $5.6 \%$. The patient did not need additional treatment, since normoglycemia was achieved on diet therapy. The patient with CEL (MODY 8) variant was diagnosed by random blood test measurement (HbA1C $8.7 \%$ ) and sulfonylurea treatment was initiated. After oneyear of oral anti-diabetic therapy, since the HbA1c value had risen to $10.8 \%$, insulin treatment was deemed necessary.

\section{Discussion}

The relative frequencies of monogenic forms of diabetes show variations according to the countries where the studies took place. For example, MODY 3 is the most common subtype in the United Kingdom, The Netherlands, Denmark, and Norway, but MODY 2 is the most common in Germany, Austria, Poland, the Czech Republic, Italy, Greece, and Spain (6). In our study, MODY 2 was the most frequently detected 
subtype (59.2\%). These differences may be explained by a variety in ethnicity and the use of different selection criteria for patients for genetic testing (6).

GCK-MODY is one of the most common subtypes of monogenic diabetes in the pediatric diabetes clinic and its clinical phenotype is remarkably homogeneous among patients (9). The patients show non-progressive mild hyperglycemia from birth. Their HbA1c is mildly elevated but usually below $7.5 \%$ (10). In agreement with recent studies, the mean HbA1c value of our GCK-MODY patients was $6.4 \%$. Because blood glucose does not deteriorate significantly over time, this subtype of monogenic diabetes is rarely associated with complications of diabetes and patients do not generally require any treatment (11). In most cases, diet alone is sufficient to achieve metabolic control (12). However, due to incompatibility with diet therapy in our cohort, oral anti-diabetic drugs (18\%) and insulin regimen $(19 \%)$ were added to the treatment of patients.

HNF1A-MODY is the most common form of monogenic diabetes that results in familial symptomatic diabetes (13). Almost $94 \%$ of the cases were diagnosed as HNF1A MODY with a symptomatic finding in recent reports. Over time, fasting hyperglycemia and osmotic symptoms (polyuria, polydipsia) present but patients rarely develop ketosis because some residual insulin secretion persists for many years (14). In our study, $29 \%$ of the patients had ketosis and the treatment consisted of insulin treatment or an oral anti-diabetic drug in addition to diet therapy in $71 \%$ of these patients. The development of chronic complications of diabetes is related to the degree of metabolic control (14). None of our patients developed complications.

GCK (MODY 2), HNF1A (MODY 3), and HNF4A (MODY 1) genes are responsible for many genetic causes of monogenic diabetes (6). The other monogenic forms of diabetes have been shown to represent together only a small proportion $(<5 \%)$ of cases, but molecular confirmation of the diagnosis prevents unnecessary insulin treatment in patients with monogenic diabetes and improves both metabolic control and quality of life (15). Sulfonylurea treatment was started for all our patients with KCJN11 variants and this treatment was continued during their follow-up. One-third of the patients with $A B C C 8$ variants continued sulfonylurea treatment alone, while all INS-MODY patients were started on insulin therapy and continued with this therapy. In a single-center study on monogenic diabetes conducted in Turkey, half of the patients with the $A B C C$ variant and the patient with the KCJN11 were receiving sulfonylurea therapy, while the patient with the INS variant was on insulin regimen (16).
Family history is crucial for requesting genetic analysis for monogenic diabetes and evidence such as diagnosis of diabetes under the age of 25 years in at least one family member, autosomal dominant inheritance pattern through at least three generations, or the existence of at least two first-degree relatives with diabetes should raise suspicion of monogenic diabetes (17). In our study, having a family member diagnosed with diabetes under the age of 25 was the most frequent reason for requesting genetic analysis $(56.2 \%)$. These prediction models show high sensitivity, although with relatively low positive predictive values that result in even higher proportions of variant-negative cases. However, use of these models leads to diagnosis of the more rare monogenic forms of diabetes and to distinguish monogenic diabetes from diabetes type 1 or type 2 , to avoid the unnecessary insulin or sulfonylurea treatment, which may severely affect the patient's health.

\section{Study Limitations}

Detection of all case data for the whole country was incomplete and naturally some findings were not declared. Moreover, laboratory and genetic tests were analyzed in different centers. There may also be bias in terms of case determination in different centers. Finally, lack of the standardized approach in the management of diabetes was one of the limitations of the study.

\section{Conclusion}

This is the first Turkish multicenter genetic study of children with monogenic diabetes. In this study we tried to determine distribution, and clinical and laboratory features of monogenic diabetes. Our study showed that random blood glucose measurement had an important place in diagnosis and the most common reason leading to genetic analysis was having a family member diagnosed with diabetes at a young age. Moreover, the study results may contribute to a better understanding of the pathogenesis of the most common subtypes of monogenic diabetes and to a more personalized approach to patients' treatment, followup, and genetic counselling.

\section{Acknowledgement}

We appreciate Pediatric Endocrinology and Diabetes Association and Erkan Sarı; Mikair Gerens and Can Celiloğlu for their contribution.

\section{Ethics}

Ethics Committee Approval: The study was approved by Gülhane Military Medical Academy, Ethics Committee at 17.02.2016 (number: 50687469-1491-191-16/1648-409). 
Informed Consent: Written informed consent was obtained from all participants or their parents/guardians.

Peer-review: Externally peer-reviewed.

\section{Authorship Contributions}

Surgical and Medical Practices: Damla Gökşen, Ediz Yeşilkaya, Samim Özen, Şükran Darcan, Concept: Damla Gökşen, Ediz Yeşilkaya, Samim Özen, Şükran Darcan, Design: Damla Gökşen, Ediz Yeşilkaya, Samim Özen, Şükran Darcan, Data Collection or Processing: Damla Gökşen, Ediz Yeşilkaya, Samim Özen, Yılmaz Kor, Erdal Eren, Özlem Korkmaz, Merih Berberoğlu, Gülay Karagüzel, Eren Er, Ayhan Abacı, Olcay Evliyaoğlu, Emine Demet Akbaş, Edip Ünal, Semih Bolu, Özlem Nalbantoğlu, Ahmet Anık, Meltem Tayfun, Muammer Büyükinan, Saygın Abalı, Gülay Can Yılmaz, Deniz Kör, Elif Söbü, Zeynep Şıklar, Recep Polat, Şükran Darcan, Analysis or Interpretation: Damla Gökşen, Ediz Yeşilkaya, Samim Özen, Şükran Darcan, Literature Search: Damla Gökşen, Ediz Yeşilkaya, Samim Özen, Eren Er, Şükran Darcan, Writing: Damla Gökşen, Ediz Yeşilkaya, Samim Özen, Eren Er, Şükran Darcan.

Financial Disclosure: The authors declared that this study received no financial support.

\section{References}

1. Hattersley AT, Greeley SAW, Polak M, Rubio-Cabezas O, Njølstad PR, Mlynarski W, Castano L, Carlsson A, Raile K, Chi DV, Ellard S, Craig ME. ISPAD Clinical Practice Consensus Guidelines 2018: the diagnosis and management of monogenic diabetes in children and adolescents. Pediatr Diabetes 2018;19(Suppl 27):47-63.

2. Tattersall RB, Fajans SS. A difference between the inheritance of classical juvenile-onset and maturity-onset type diabetes of young people. Diabetes 1975;24:44-53.

3. Ledermann HM. Maturity-onset diabetes of the young (MODY) at least ten times more common in Europe than previously assumed? Diabetologia 1995;38:1482.

4. Gardner DS, Tai ES. Clinical features and treatment of maturity onset diabetes of the young (MODY). Diabetes Metab Syndr Obes 2012;5:101 108.

5. Firdous P, Nissar K, Ali S, Ganai BA, Shabir U, Hassan T, Masoodi SR. Genetic testing of maturity-onset diabetes of the young current status and future perspectives. Front Endocrinol (Lausanne) 2018;9:253.

6. Kleinberger JW, Pollin TI. Undiagnosed MODY: Time for Action. Curr Diab Rep 2015;15:110.
7. Kopanos C, Tsiolkas V, Kouris A, Chapple CE, Albarca Aguilera M, Meyer R, Meyer R, Massouras A. VarSome: the human genomic variant search engine. Bioinformatics 2019;35:1978-1980.

8. Richards S, Aziz N, Bale S, Bick D, Das S, Gastier-Foster J, Grody WW, Hegde M, Lyon E, Spector E, Voelkerding K, Rehm HL; ACMG Laboratory Quality Assurance Committee; ACMG Laboratory Quality Assurance Committee. Standards and guidelines for the interpretation of sequence variants: a joint consensus recommendation of the American College of Medical Genetics and Genomics and the Association for Molecular Pathology. Genet Med 2015;17:405-424. Epub 2015 Mar 5

9. Prisco F, Iafusco D, Franzese A, Sulli N, Barbetti F. MODY 2 presenting as neonatal hyperglycaemia: a need to reshape the definition of “neonatal diabetes"? Diabetologia 2000;43:1331-1332.

10. Steele AM, Wensley KJ, Ellard S, Murphy R, Shepherd M, Colclough K, Hattersley AT, Shields BM. Use of HbA1c in the Identification of Patients with Hyperglycaemia Caused by a Glucokinase Mutation: Observational Case Control Studies. Lin X (Erick), editor. PLoS ONE 2013;8:e65326.

11. Stride A, Shields B, Gill-Carey O, Chakera AJ, Colclough K, Ellard S, Hattersley AT. Cross-sectional and longitudinal studies suggest pharmacological treatment used in patients with glucokinase mutations does not alter glycaemia. Diabetologia 2014;57:54-56. Epub 2013 Oct 4

12. Osbak KK, Colclough K, Saint-Martin C, Beer NL, Bellanné-Chantelot C, Ellard S, Gloyn AL. Update on mutations in glucokinase (GCK), which cause maturity-onset diabetes of the young, permanent neonatal diabetes, and hyperinsulinemic hypoglycemia. Hum Mutat 2009;30:1512-1526.

13. Pearson ER, Pruhova S, Tack CJ, Johansen A, Castleden HAJ, Lumb PJ, Wierzbicki AS, Clark PM, Lebl J, Pedersen O, Ellard S, Hansen T, Hattersley AT. Molecular genetics and phenotypic characteristics of MODY caused by hepatocyte nuclear factor 4alpha mutations in a large European collection. Diabetologia 2005;48:878-885. Epub 2005 Apr 14

14. Isomaa B, Henricsson M, Lehto M, Forsblom C, Karanko S, Sarelin L, Häggblom M, Groop L. Chronic diabetic complications in patients with MODY3 diabetes. Diabetologia 1998;41:467-473.

15. Kim S-H. Maturity-onset diabetes of the young: what do clinicians need to know? Diabetes Metab J 2015;39:468-477.

16. Şıklar Z, de Franco E, Johnson M, Flanagan S, Ellard S, Ceylaner S, Boztuğ K, Doğu F, İkincioğulları A, Kuloğlu Z, Kansu A, Berberoğlu M. Monogenic diabetes not caused by mutations in mody genes: a very heterogenous group of diabetes. Exp Clin Endocrinol Diabetes 2018;126:612-618. Epub 2017 Nov 28

17. Tattersall RB. Mild familial diabetes with dominant inheritance. Q J Med 1974;43:339-357. 